\title{
Evaluación de los estadíos de maduración esquelética según Fishman en niños de 8 a 16 años de edad del distrito de Tambo de Mora-Chincha
}

\section{Oswaldo Ramos Portocarrero 1 Abraham Meneses López ${ }^{2}$}

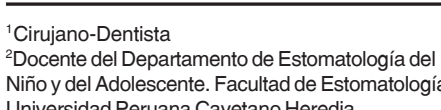

Niño y del Adolescente. Facultad de Estomatología. Universidad Peruana Cayetano Heredia

\section{Correspondencia}

Oswaldo Ramos Portocarrero, Las Esmeraldas 233, Lima 13 - Perú. Telf: 4722799.

E-mail : osramport@yahoo.com

Aceptado para publicación: 08 de abril del 2005
Ramos O, Meneses A. Evaluación de los estadios de maduración esquelética según Fishman en niños de 8 a 16 años de edad del distrito de Tambo de Mora-Chincha. Rev Estomatol Herediana 2005; 15(1): 5 - 10

\section{RESUMEN}

El propósito del estudio fue determinar el momento de aparición de los estadíos de maduración esquelética en niños peruanos entre 8 y 16 años de edad del distrito de Tambo de Mora-Chincha. Fueron tomadas radiografías carpales a 180 sujetos (90 varones y 90 mujeres). Se subagruparon en número de 10 sujetos por edad y sexo. Se utilizó el método de Fishman para determinar el momento de aparición de cada estadío. El diseño del estudio fue descriptivo y transversal. Se utilizó la prueba estadística de Wilcoxon y el test t de student para la comparación. La edad promedio para el inicio de la curva de crecimiento fue de 9,18 años en varones y 8,00 años en mujeres. El Pico Máximo de Velocidad de Crecimiento Puberal (PMVCP) en mujeres, fue a menor edad que en los varones; la edad promedio para el estadío 6 (PMVCP en mujeres) fue a los 10,83 años, a diferencia de los varones donde la edad promedio para el estadío 7 (PMVCP en varones) fue de 13,95 años. Existió una alta correlación entre estadío de maduración esquelética y estatura, como también estadío de maduración esquelética y edad, ( $\mathrm{p}<0.001)$.

Palabras clave: DESARROLLO ÓSEO. crecimiento \& desarrollo / ADOLESCENTE.

Evaluation of the skeletal maturation stages, according to Fishman, in Peruvian children between the ages of 8 and 16 years old from the districts of Tambo de Mora in Chincha

ABSTRACT

The purpose of the study was to determine the moment when the skeletal maturation stages appear in Peruvian children between the ages of 8 and 16 years old from the district of Tambo de Mora in Chincha. Hand wrists radiographs were performed on 180 subjects (90 males and 90 females). The sample was divided in groups of 10 according to age and sex. The Fishman method was used to determine the moment each stage appears. The design of the study was descriptive and transversal. The statistical tests used were the Student t-test which compares averages by independent samples according to sex and the Wilcoxon Range for comparison of ages among the groups. The average age at the beginning of the growth spurt was 9,18 years in males and 8,00 years in females. The Maximum Velocity Peak of Puberty Growth (MVPPG) in females was at an earlier age than in males. The average age for stage 6 (MVPPG in females) was at 10,83 years unlike the average age for stage 7 (MVPPG in males) which was at 13,95 years. The average of the skeletal maturation stage in females was 1,64 years before than in males; there are significant differences between average stature for each Fishman stage by sex mainly at the final stages $(6,7,8,9,10)$, where males presented greater average stature than females. A high correlation was observed between the skeletal maturation stage and stature, as well as between skeletal maturation stage and age $(\mathrm{p}<0.001)$.

Key words: BONE DEVELOPMENT. growth \& development / ADOLESCENT.

\section{Introducción}

Diversos métodos para evaluar los indicadores de maduración esquelética han demostrado la validez de las radiografías de mano y muñeca para graficar la curva de crecimiento en diferentes grupos poblacionales. Estos métodos relacionan la maduración ósea con factores tales como edad cronológica, talla, calcificación de dientes permanentes, menarquia, aparición de los caracteres sexuales secundarios, grupo étnico, sexo, medio ambiente, herencia, en- tre otros (1-7).

Uno de los métodos más seguros para determinar el desarrollo físico puede ser hecho mediante el examen de las estructuras calcificadas de la mano y muñeca, en los cuales el grado de maduración de los huesos individuales puede ser estimado, y la edad esquelética se puede determinar comparándolo con estándares radiográficos. Entre ellos, uno de los más usados por su confiabilidad y facilidad de aplicación es el método de Fishman (8).
La evaluación radiológica de las placas terminales epifisiarias proporciona una indicación exacta de la edad ósea para la comparación con la edad cronológica y permite predecir el potencial para posteriores aumentos de la estatura $(9,10)$.

Teóricamente, cualquiera de las partes del cuerpo puede ser empleada en la valoración de la edad ósea, pero en la práctica, la mano y el carpo son utilizadas especialmente después del primer año de vida debido a que poseen un 
gran número de huesos y epífisis en desarrollo por lo cual resulta posible el seguimiento de los cambios a través del tiempo, a medida que el individuo crece. Además se facilita su examen radiográfico con mínima exposición radiológica al resto del cuerpo (3). La determinación de la edad ósea ha sido el método más utilizado para la evaluación de la maduración (11-14), consiste en la medición del avance del desarrollo óseo a través del análisis radiográfico.

La secuencia para cada hueso es la misma en cuanto a los eventos que en él ocurrirán, independientemente del grado de retraso o adelanto con respecto a la edad cronológica $(11,12,15-18)$. La osificación de los huesos carpales se grafica en las curvas de velocidad de incremento estatural $(4,11,14-19)$, la cual tiene cuatro partes reconocibles $(17,18)$ :

- Inicio de la curva incremental de crecimiento.

- Aceleración del crecimiento puberal.

- Pico de crecimiento puberal.

- Término o final del crecimiento puberal.

Los huesos carpales fueron identificados por Lyser en 1683, quien los describió como huesos cortos, esponjosos, envueltos en una fina capa de hueso compacto $(20,21)$.

En 1759 el Conde Philibert Gueneau De Montbeillard, realizó en su hijo la primera investigación longitudinal sobre crecimiento desde el nacimiento hasta los 18 años para determinar el incremento anual de su estatura, graficando así, la primera curva de crecimiento e identificando los diferentes picos del incremento estatural $(13,22)$.

Ranke en 1896, fue el primero en estudiar radiografías de mano y muñeca para observar el desarrollo de las estructuras óseas $(5,20)$.

En 1912, Boas relacionó los estadios de desarrollo óseo con los diferentes indicadores de crecimiento puberal y edad cronológica, concluyendo que los huesos carpales pueden ser usados como indicadores del crecimiento físico general del individuo (6).

Helm en 1928, demostró que la osificación de los huesos de la mano ocurría a los 12,5 años promedio en varones y que además coincidía con el inicio de la adolescencia $(4,6)$.

Greulich y Pyle (10) en 1950 publi- can "Radiographic atlas of skeletal development of de hand and wrist” posteriormente revisado y vuelto a publicar en 1959 y 1972; el método propone determinar la edad ósea comparando imágenes radiográficas con respecto a un patrón $(12,14,15)$.

Mackay en 1952, estudió una muestra de 602 niñas y 758 niños de una tribu de origen Bantú (Australianos), realizando comparaciones mensuales de radiografías carpales y demostrando que el orden de aparición de los sitios de osificación era similar entre las diferentes poblaciones y también similar a lo encontrado por Flory et al. (6,23) en 1936 en una población anglosajona. Bjork y Helm (7) en 1967, crearon un método para predecir el Pico de Máximo Crecimiento Estatural (PMCE).

Fishman (8) en 1982, en su estudio demostró que la radiografía de mano podía dar información del crecimiento en relación al estado de maduración con importantes aplicaciones clínicas en el diagnóstico ortodóncico.

Kopecky y Fishman (24), en 1993, demostraron que los efectos ortopédicos más significativos para redireccionar el crecimiento mandibular, eran producidos alrededor del pico máximo del crecimiento puberal.

Los estudios longitudinales de Hägg y Taranger $(11,14,18)$, en 1980 y 1982, sobre picos de crecimiento, caracteres sexuales secundarios y erupción dentaria en niños suecos demostraron que en promedio, la curva de crecimiento puberal comenzó a los 10 años en mujeres y 12,1 años en varones y terminó a los 14,8 años en niñas y 17,1 en niños.

Tiberio (4), en 1988, realizó un estudio en maduración esquelética en niños brasileños (blancos) de 8 a 15 años, concluyó que el inicio del crecimiento puberal fue entre los 10 y 11 años en niñas y entre los 11 y 12 años en los niños, registrando como el pico de crecimiento en niños entre los 12 y 13 años y entre los 15 y 16 años para los niños.

Numerosos son los factores que pueden ejercer influencia en los procesos de maduración a través de mecanismo conocidos y otros aún no precisados $(4,15,16,22)$, se distinguen cuatro grandes grupos que comprenderían la mayor parte de estos factores: genéti-cos, hormonales, nutricionales y patológicos $(2,3)$.
Método de Fishman para evaluar los indicadores de maduración esquelética (17)

Los cambios óseos vistos en la mano y muñeca son indicadores de cambios esqueléticos generales. El concepto de edad esquelética basado en la radiografía carpal ha sido desarrollado de modo que pueda ser comparado con la edad cronológica del individuo $(2,17,18)$.

El método de Fishman (17) (1979) estuvo en desarrollo por más de cuatro años resultando un sistema válido en situaciones clínicas y de investigación. La secuencia de cuatro indicadores de osificación progresa a través del ensanchamiento epifisiario en las falanges, la osificación del sesamoideo abductor del pulgar, el capeamiento (cubrimiento), de las epífisis sobre sus respectivas diáfisis, y la fusión de las epífisis con sus diáfisis. La secuencia de aparición de los 11 Estadíos de Maduración Esquelética (EME) es excepcionalmente estable. El estudio reportó sólo tres desviaciones en más de dos mil observaciones, y éstas no afectaron las interpretaciones respectivas (Fig. 1).

Estudios previos, realizados en diversas partes del país, tales como Hi

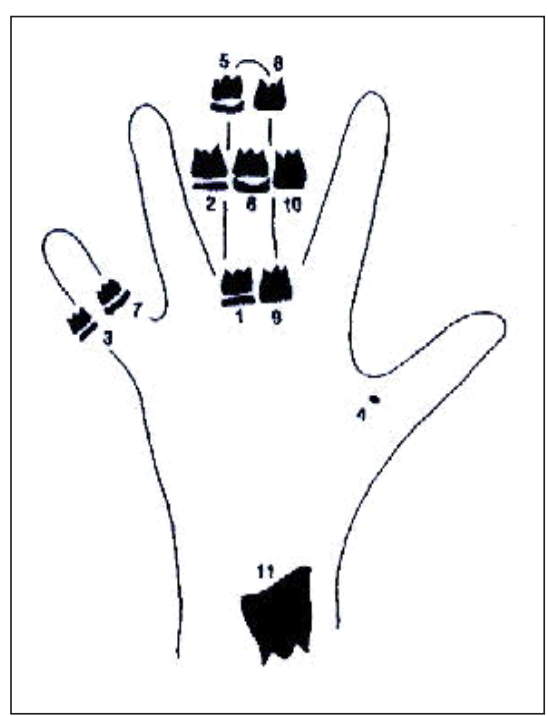

Figura 1. Indicadores de maduración esquelética según fishman (1982). Ancho de la epífisis tan igual como su diáfisis: (1)Tercer dedo - falange proximal. (2)Tercer dedo - falange media. (3)Quinto dedo - falange media; calcificación del sesamoideo abductor del pulgar. (4)Sesamoideo cubrimiento. (5)Tercer dedo - falange distal. (6)Tercer dedo - falange media. (7)Quinto dedo - falange media; Fusión de epífisis y diáfisis. (8)Tercer dedo - falange distal; (9)Tercer dedo - falange proximal. (10)Tercer dedo falange media. (11)Radio. 
dalgo (25) (1999), Meneses (26) (2002) y Raymundo (27) (2004) han demostrado que el pico de velocidad de crecimiento en adolescentes, esta bastante relacionado con ciertos eventos, los cuales ocurren durante el curso de desarrollo de la mano y muñeca.

El presente estudio es parte de una línea de investigación y pretende determinar el momento de aparición de los indicadores de maduración esquelética en niños peruanos entre 8 y 16 años de edad del distrito de Tambo de Mora - Chincha, quienes tienen una fuerte ascendencia de raza negra con un biotipo particular y condiciones alimenticias casi exclusivamente a base de pescados y mariscos por ser un puerto marítimo del sur chico.

\section{Materiales y método}

El diseño del estudio fue de tipo descriptivo y transversal. Para la muestra se seleccionaron 180 sujetos entre 8 y 16 años de edad, residentes en el Distrito de Tambo de Mora, Provincia de
Chincha, Departamento de Ica, cumpliendose las siguientes consideraciones éticas: La presente investigación fue registrada y aprobada por el Comité Institucional de Ética de la Universidad Peruana Cayetano Heredia

La ejecución del presente trabajo se realizó previa entrega y aceptación del consentimiento informado por escrito a cada autoridad, apoderado y/o padre de familia.

Asimismo se cumplieron los siguientes criterios de selección: peruanos de nacimiento, con ambos padres peruanos, residentes en el mencionado distrito, en estado nutricional normal, que no presentaran o hayan presentado algún síndrome o trastorno sistémico de tipo endocrinológico, que no presentaran historia de traumatismo en la región carpal, que no presentaran historia de intervenciones quirúrgicas por alteraciones sistémicas de cualquier etiología. Se tomaron radiografías carpales de la mano y muñeca izquierda, nítidas y de buen contraste. Finalmente se obtuvo un tamaño muestral de 90 sujetos para cada sexo la cual a su vez fue subagrupa-da en nueve subgrupos de acuerdo a la edad.

Los datos se registraron en un cuadro general (matriz) para cada sujeto según sexo.

Los datos fueron analizados mediante pruebas univariadas de distribución de frecuencias y determinaron porcentajes. Adicionalmente se empleó la media aritmética y la desviación estándar para describir las variables talla y edad. Prueba bivariada paramétrica t de Student, para comparar promedios, por muestras independientes y suma de rangos de Wilcoxon y U de Mann Withney la cuales compararon las variables edad y talla para cada estadío de maduración según sexo. Finalmente se empleó el coeficiente de correlación de Pearson para determinar la asociación lineal entre las variables edad y talla con respecto a los estadíos de maduración.

Tabla 1. Estadío de Fishman prevalente en cada edad según sexo.

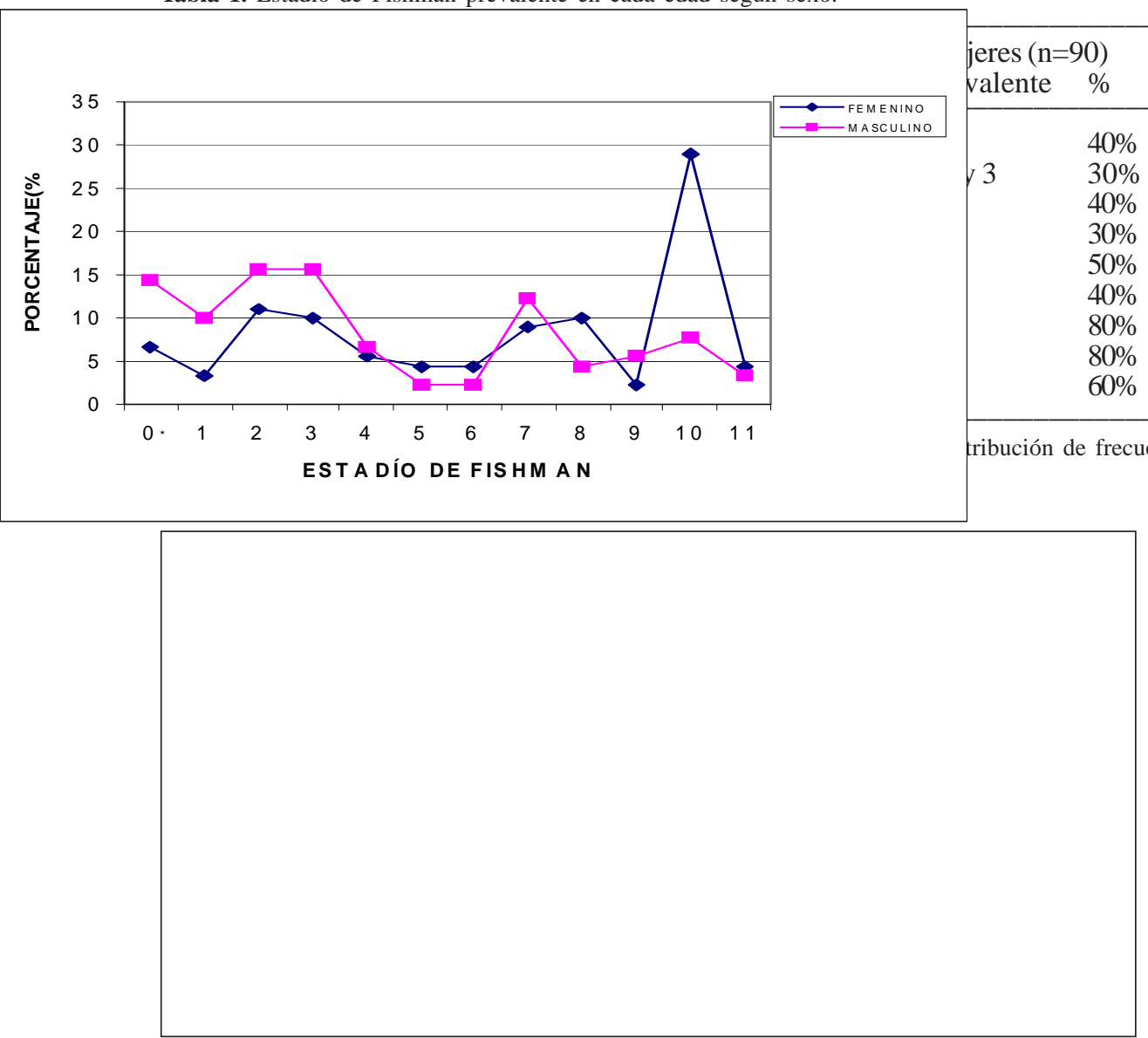

Figura 2. Comparación de la distribución de frecuencias de los estadios de Fishman por sexo.

\section{Resultados}

En la Tabla 1, se aprecia una alta prevalencia de niños que aún no inician la curva de crecimiento prepuberal, dicha condición fue registrada con el número “0” para su figuración en la tabla, aún cuando no está considerado entre los estadíos de Fishman. A su vez a los 8, 9, 10 y 16 años no se encontraron diferencias estadísticamente significativas $(p>0,05)$ entre sexos, pero sí estadíos inferiores prevalentes de los varones con respecto a las mujeres en la mayoría de los casos.

En la Figura 2, en donde se muestra 
que los varones en un mayor porcentaje respecto a las mujeres alcanzan los primeros 4 estadíos al igual que para el estadío 7 y 9 a diferencia de los estadíos 5, 6, 8, 10 y 11 donde las mujeres alcanzan mayor porcentaje respecto a los varones, esto se evidencia principalmente en el estadío 10 donde el porcentaje alcanzado por las mujeres es del $28,89 \%$ comparado con el de los varones de 7,78\% para dicho estadío.

En la Tabla 2 se establecieron los rangos promedio de edad para el momento de aparición de cada estadío de Fishman en ambos sexos. Para el estadío que corresponde al pico máximo de velocidad de crecimiento puberal (PMVCP) señalado por Fishman, se encontró que en las mujeres, sucede a menor edad que en los varones; la edad promedio para el estadío 6 (PMVCP en mujeres) se encuentra a los 10,83 años ( $\mathrm{p}=0,015)$, a diferencia de los varones donde la edad promedio para el estadío 7 (PMVCP en varones) es de 13,95 años ( $\mathrm{p}=0,002)$. Como se observa en la tabla se encontraron diferencias significativas entre las edades promedio de ambos sexos y la aparición de los estadíos 2, 3, 5, 6 de Fishman y una diferencia altamente significativa a nivel de los estadíos 4, 7, 8 y 10; lo cual evidencia una vez más que los estadíos de Fishman se presentan a una menor edad en las mujeres comparado con los varones.

En la Tabla 3 (Fig. 3), en el grupo de los varones podemos observar un incremento significativo en la estatura de aproximadamente $10 \mathrm{~cm}$. entre los EME 1-3 y 5-7. En el caso de las mujeres se observaron incrementos importantes de estatura entre los estadíos 1-3 (aproximadamente $10 \mathrm{~cm}$.) y 6-8 (aproximadamente $12 \mathrm{~cm}$.). Además se observan diferencias estadísticas significativas en la estatura a nivel de los estadíos 4, 5, 6 y 9; y una diferencia estadística altamente significativa en la estatura a nivel de los estadíos 7, 8 y 10 según sexos $(\mathrm{p}<0,05)$. Se evidencia también que no existen diferencias estadísticamente significativas en cuanto a la estatura a nivel de los primeros tres estadíos, mientras que sí lo hay en los últimos estadíos lo cual indicaría que el aumento estatural en los varones se da básica
Tabla 2. Comparación de la edad promedio de aparición de cada estadío de fishman según sexo.

\begin{tabular}{|c|c|c|c|c|c|}
\hline \multirow[b]{2}{*}{ Estadío } & \multicolumn{2}{|c|}{ Varones $(\mathrm{n}=90)$} & \multicolumn{3}{|c|}{ Mujeres (n=90) } \\
\hline & Edad & $\mathrm{DE}$ & Edad & $\mathrm{DE}$ & $\mathrm{p}$ \\
\hline 0 & 8.88 & 1,20 & 8,39 & 0,80 & 0,535 \\
\hline 1 & 9,18 & 1,03 & 8,00 & 0,01 & 0,091 \\
\hline 2 & 9,98 & 1,57 & 8,75 & 1,15 & 0,016 \\
\hline 3 & 10,68 & 1,54 & 9,06 & 0,72 & 0,019 \\
\hline 4 & 12,39 & 0,81 & 9,89 & 0,80 & 0,003 \\
\hline 5 & 13,53 & 0,70 & 9,58 & 0,61 & 0,034 \\
\hline 6 & 13,55 & 0,74 & 10,83 & 0,49 & 0,015 \\
\hline 7 & 13,95 & 1,29 & 11,80 & 0,70 & 0,002 \\
\hline 8 & 14,79 & 0,51 & 12,94 & 1,45 & 0,005 \\
\hline 9 & 14,82 & 1,08 & 14,02 & 1,39 & 1,001 \\
\hline 10 & 14,63 & 0,97 & 14,28 & 0,99 & 0,002 \\
\hline 11 & 15,72 & 2,61 & 14,83 & 1,23 & 0,413 \\
\hline
\end{tabular}

$\mathrm{DE}=$ desviación estandar

Tabla 3. Comparación de la estatura promedio para cada estadío de Fishman según sexo.

\begin{tabular}{|c|c|c|c|c|c|}
\hline \multirow[b]{2}{*}{ Estadío } & \multicolumn{2}{|c|}{ Varones $(\mathrm{n}=90)$} & \multicolumn{3}{|c|}{ Mujeres (n=90) } \\
\hline & Edad & $\mathrm{DE}$ & Edad & $\mathrm{DE}$ & $\mathrm{p}$ \\
\hline $0 *$ & 127,38 & 0,07 & 124,50 & 0,08 & 0,777 \\
\hline 1 & 130,89 & 0,06 & 121,00 & 0,03 & 0,145 \\
\hline 2 & 134,93 & 0,07 & 128,60 & 0,10 & 0,120 \\
\hline 3 & 140,86 & 0,09 & 131,22 & 0,06 & 0,149 \\
\hline 4 & 147,17 & 0,06 & 137,60 & 0,03 & 0,042 \\
\hline 5 & 152,50 & 0,05 & 141,25 & 0,04 & 0,032 \\
\hline 6 & 155,50 & 0,13 & 139,25 & 0,05 & 0,014 \\
\hline 7 & 162,73 & 0,07 & 148,13 & 0,06 & 0,003 \\
\hline 8 & 159,50 & 0,05 & 151,56 & 0,07 & 0,003 \\
\hline 9 & 166,60 & 0,05 & 151,00 & 0,07 & 0,042 \\
\hline 10 & 160,43 & 0,06 & 152,31 & 0,04 & 0,001 \\
\hline 11 & 165,00 & 0,05 & 151,75 & 0,02 & 0,066 \\
\hline
\end{tabular}

$\mathrm{DE}=$ desviación estandar

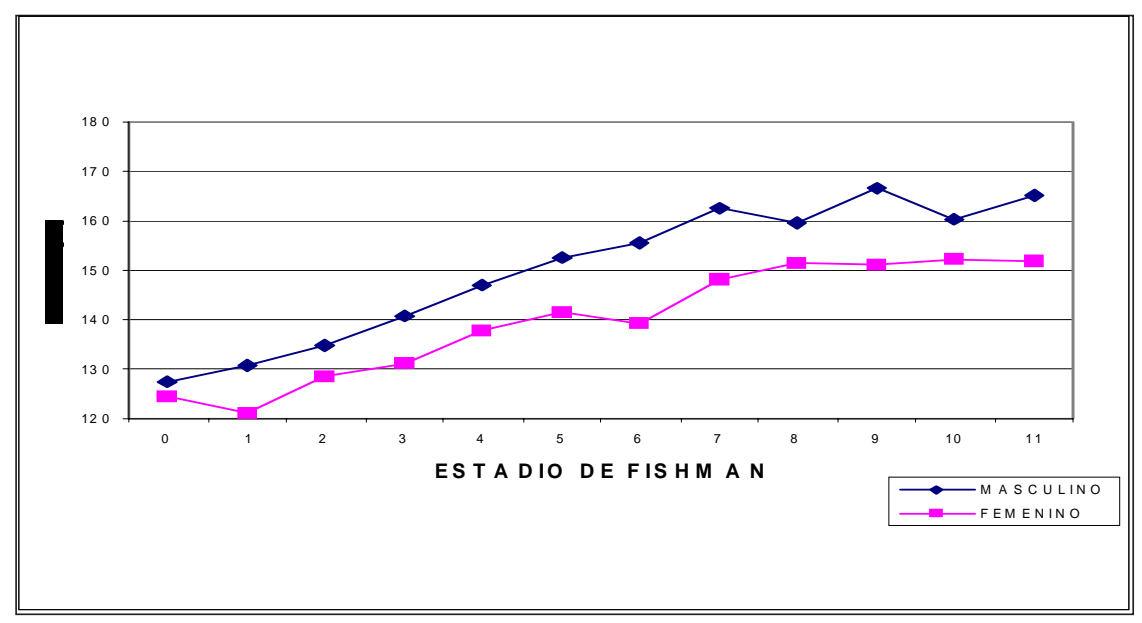

Figura 3. Comparación de las curvas de crecimiento por sexos. 
mente al final de los estadíos de Fishman.

Finalmente la correlación existente entre los EME y la talla según sexo fue muy similar ( 0,872 y 0,86 para varones y mujeres respectivamente) y estadísticamente diferentes de cero $(p<0,001)$. Del mismo modo la correlación existente entre los EME y la edad fue de 0,875 para varones y de 0,922 para mujeres, siendo estadísticamente diferente de cero en ambos casos $(\mathrm{p}<0,001)$.

\section{Discusión}

En nuestro país las condiciones alimenticias son diversas y muchas veces relacionadas a las condiciones geográficas, las cuales son en algunos casos muy particulares dependiendo de cada región o población. El distrito de Tambo de Mora, es un puerto marítimo ubicado en la provincia de Chincha departamento de Ica, cuyos pobladores tienen una alimentación a base de pescados y mariscos; la ocupación del 90\% de la población está relacionada a la pesca o el trabajo como operarios en la fábricas de harina de pescado. La comparación de las estaturas promedio, muestran un patrón uniforme de distribución. Así se puede apreciar que las niñas presentan mayor estatura que los varones a los 9 años de edad, ésto puede explicarse por el hecho que las mujeres a esta edad ya han iniciado la Curva de Crecimiento Puberal mientras que la mayoría de los varones, aún no inician dicha curva (Tabla 2). Dicho aspecto está asociado a los cambios hormonales prepuberales, específicamente la adrenarquia, secreción importante de andrógenos que estimula el aumento estatural, el cual se da en un primer momento a los 6 y 7 años en las niñas y entre los 7 y 8 años en los niños (aproximadamente un año antes del incremento de la estatura, en estudios de población nacional y escandinava) (28, 29). Un segundo incremento importante de andrógenos ocurre a los 10 años en las niñas y 12 años en los varones, y precede también al pico de máxima velocidad de crecimiento puberal (PMVCP), ello explica porqué las niñas, presentaron mayor estatura que los varones a los 12 años de edad y los varones, presentaron mayor estatura que las mujeres, en- tre los 14 y 16 años de edad. Estos incrementos de altura corresponden a los EME 7 (PMVCP) de Fishman $(8,30)$ para ambos casos (Tabla 1, Fig. 2).

Se observa también que para nuestra muestra, el estadío 6 de Fishman (PMVCP), en mujeres ocurre a los 10,83 años, para los varones el estadío 7 de Fishman (PMVCP) es a los 13,95 años.

Grave (13) encontró el PMVCP en niñas a los 11,8 años y en los varones a los 13,8 años de edad. Hägg y Taranger (31) en niños suecos encontraron que el PMVCP a los 12 años en niñas y a los 14 años de edad en los varones. Fishman (17), encontró resultados similares a este último estudio.

Hidalgo (25) (1999), en una muestra peruana, realizado en la ciudad de Lima, encontró que el PMVCP, en mujeres ocurre a los 11,11 años (EME 6), y en los varones el PMVCP (EME 7), a los 13,2 años de edad. Como se puede notar el PMVCP se presenta a edades muy similares comparado con los resultados de nuestra muestra.

Meneses (26) (2002), en un estudio realizado en la ciudad de Lima, en mujeres y varones con nutrición normal, encontró que el PMVCP (EME 6 de Fishman) para las mujeres ocurre a los 10,91 años, y para los varones el PMVCP (EME 7 de Fishman) se da a los 14,23 años, mostrando valores muy similares a los encontrados en el presente estudio.

Raymundo (27) (2004), en un estudio realizado en la ciudad de Cerro de Pasco, en mujeres y varones con nutrición normal, encontró que el PMVCP (EME 6 DE Fishman) fue de 12,58 años para las mujeres y para los varones el PMVCP (EME 7 de Fishman) se dio a los 15,57 años, aunque dicho estudio fue realizado en la altura, nos muestra también claramente que el PMVCP siempre se da antes en las mujeres.

Las mujeres, presentaron estadíos más avanzados que sus correspondientes pares varones en un rango promedio de 1,64 años (Tabla 2).

Bjork, Helm, Moore y Glendon, coinciden en que el pico de crecimiento craneofacial se sitúa en un período que varía entre 6 a 12 meses después del
PMVCP. Fishman (8, 30), afirma que está un estadío por delante del PMVCP, es decir en las mujeres en el estadío 7, y en los varones en el estadío 8; el conocimiento de ello permitirá la planificación adecuada de tratamientos ortopédicos que impliquen estimular o redireccionar el crecimiento craneofacial.

\section{Conclusiones}

- Se encontraron diferencias en las edades promedio de aparición de cada estadío de Fishman según sexo, a excepción de los estadíos 1 , 9 y 11.

- Los estadíos de maduración een las mujeres se presentaron 1,64 años promedio antes que en varones.

- El pico máximo de la velocidad de crecimiento puberal promedio se presentó a los 10,83 años en las mujeres (EF 6) y a los 13,95 años para los varones (EF 7).

\section{Referencias bibliográficas}

1. Krogman W. The meaningful interpretation of growth and growth data by the clinician. Am J Orthod 1958; 44:411-32.

2. Chapman SM. Ossification of the adductor sesamoid and the adolescent growth spurt. Am J Orthod 1972; 42;236-44.

3. Gabriel R, Tenenbaum J. Determinación práctica de la edad carpal, ortodoncia. Editorial ateneo de ortodoncia y ortopedia funcional de los maxilares del círculo de odontología de misiones. 1979; 85:519.

4. Tiberio SO. Estudio de maturacao esquelética de criancas brasileiras leucodermás de 8 a 15 años, em referencia a ossificacao do ossos pisiforme, ganchoso, falanges media e proximal dos dedos 2 e 3 . Instituto metodista do ensino superior sao bernado do campo. 1988.

5. Caffey J. Pediatric X-ray diagnosis. 6ta. ed; Chicago. Year Book Medical Publisher Inc. 1972.

6. Bergersen EO. The male adolescent facial growth spurt: It's prediction and relation to skeletal maturation. Angle Orthod 1972; 42:319-38. 
7. Bjork A, Helm S. Prediction for the age of maximum puberal growth in the body height. Angle Orthod 1967;37:134-43.

8. Fishman L. Radiografic evaluation for skeletal maturation. A clinical oriented method based on hand - wrist films. Angle Orthod 1982;52:182-9.

9. West JB. Bases fisiológicas de la práctica médica. Editorial Panamericana. Buenos Aires. 1993.

10. Greulich WW, Pyle SE. Radiographic atlas of skeletal development of the hand and wrist. Stanford, CA: Stanford University Press; 1959.

11. Hägg U, Taranger J. Maduration indicators and the puberal growth spurt. Angle Orthod 1979;49 (3):1819.

12. Grave K, Brown T. Carpal radiographs in orthodontic treatment. Am J Orthod 1979; 75(1):27-45.

13. Grave K, Brown T. Skeletal ossification and the adolescent growth spurt. Am J Orthod 1976;69(6):611-9.

14. Hägg U, Taranger J. Menarche and voice change as indicators of the puberal growth supurt. Acta Odontol Scand 1980;38: 179-86.

15. Falkner F. Evaluación del crecimiento. Centro de investigación endocrinológica. Buenos Aires;1986.

16. Hathaway W, Hay Groothuis J, Paisley J. Diagnóstico y tratamiento pediátricos. México DF: Editorial El Manual Moderno;1995.
17. Fishman L. Chronological versus skeletal age, an evaluation of craniofacial growth. Angle Orthod 1979; 49(3):181-9.

18. Hägg U, Taranger J. Skeletal stages of the hand and wrist as indicators of the puberal growth spurt. Acta Odontol Scand 1980; 38:187-200.

19. Ten Cate A. Histología oral, desarrollo, estructura y función. Buenos Aires: Editorial Panamericana; 1986.

20. Enciclopedia británica. Enciclopedia británica Inc. 1982. vol. 30; p. 457.

21. Valverde HR. Correlación entre estadíos de calcificación de caninos y segundas premolares mandibulares con la curva de crecimiento puberal maxilar y mandibular. [tesis de especialista]. Lima: Universidad Peruana Cayetano Heredia; 2000.

22. Pryor JM. Difference in the time of development of centers of ossification in the male and female skeleton. Anat Rec 1923; 25:257-73.

23. Manzaneda J. Maduración ósea, método TW2. [Seminario]. Universidad Peruana Cayetano Heredia, Facultad de Estomatología. 1992.

24. Kopecky GR, Fishman LS. Timing of cervical headgear treatment based on skeletal maturation. Am J Orthod Dentofac Orthop 1993;104: 162-9.

25. Hidalgo C. Determinación de la curva de crecimiento según fishman en peruanos de 9 a 16 años de edad. [tesis de especialista]. Lima: Universidad Peruana Cayetano Heredia;1999.

26. Meneses A. Influencia de la altura geográfica y el estado nutricional sobre los indicadores de maduración esquelética en peruanos de 8 a 16 años de edad. [tesis doctoral]. Lima: Universidad Peruana Cayetano Heredia; 2002.

27. Raymundo J. Asociación entre estado nutricional y estadíos de maduración esquelética según el índice de Fishman en escolares de 8 a 16 años de edad en la ciudad de Cerro de Pasco. [tesis de especialista]. Lima: Universidad Peruana Cayetano Heredia;2004.

28. Gonzáles GF, Carrillo C, Villena A, Alarcón I. Factores biomédicos que determinan la fertilidad en la altura. Acta Andina 1993; 2:141-59.

29. Pirinen S. Endocrine regulation of craneofacial growth. Acta Odont Scan 195;53:179-185.

30. Fishman L. Can cephalometric X-rays of the cervical column be used instead of hand-wrist $\mathrm{x}$-rays to determine a patient's maturational age? Am J Orthod Dentofac Orthop 2002;122 (1):18A-19A.

31. Hägg U, Taranger J. Maturation indicators and the puberal growth spurt. Am Journal of Orthod and Dentofac Orthop. 1982;82(4):2 